\title{
Identification of an Alveolar Macrophage-Related Core Gene Set in Acute Respiratory Distress Syndrome
}

\author{
Chunling Zhao',* \\ Jingjia $\mathrm{Mo}^{2}$,* \\ Xiaowen Zheng' \\ Zimeng $\mathrm{Wu}^{\prime}$ \\ Qian Li ${ }^{1}$ \\ Jihua Feng' \\ Jiefeng Luo' \\ Junyu $\mathrm{Lu}^{3}$ \\ Jianfeng Zhang (D)
}

'Department of Emergency Medicine, The Second Affiliated Hospital of Guangxi Medical University, Nanning, 530007, People's Republic of China; ${ }^{2}$ Department of General Medicine, The Second Affiliated Hospital of Guangxi Medical University, Nanning, 530007, People's Republic of China; ${ }^{3}$ Intensive Care Unit, The Second Affiliated Hospital of Guangxi Medical University, Nanning, 530007, People's Republic of China

*These authors contributed equally to this work
Purpose: Acute respiratory distress syndrome (ARDS) is a rapidly progressive diffuse lung injury that is characterized by high mortality and acute onset. The pathological mechanisms of ARDS are still unclear. But alveolar macrophages have been shown to play an important role in inflammatory responses during ARDS. We aimed to find the biomarkers for ARDS for early diagnosis, to give ARDS patients timely treatment.

Methods: Gene expression profiles were downloaded from Gene Expression Omnibus (GEO) and screened for differentially expressed genes (DEGs). The common upregulated genes in all the datasets were defined as circulating ARDS alveolar macrophage-related genes (cARDSAMGs). We performed a functional enrichment analysis to explore potential biological functions of cARDSAMGs, and we built protein-protein interaction networks. Gene set variation analysis (GSVA) was used to calculate the core gene set variation analysis (CGSVA) score for individual samples. Receiver operating characteristic (ROC) curve analysis was applied on the CGSVA score to evaluate its ability for diagnosis of ARDS.

Results: A total of 60 genes were upregulated in all ARDS datasets and were therefore denominated as cARDSAMGs. The cARDSAMGs were significantly involved in multiple inflammation-, immunity- and phagocytosis-related biological processes and pathways. In the protein-protein interaction network associated with host responses to ADRS, eight genes were identified as a core gene set: PTCRA, JAG1, C1QB, ADAM17, C1QA, MMP9, VSIG4 and TNFAIP3. ROC curve analysis showed that the CGSVA score may be considered as a biomarker for ARDS: it was significantly higher in patients with ARDS than those in healthy in both alveolar lavage fluid and whole blood.

Conclusion: The ARDS alveolar macrophage-related CGSVA score may be useful as a biomarker for ARDS.

Keywords: acute respiratory distress syndrome, lung injury, gene expression omnibus, differentially expressed genes

\section{Introduction}

Sepsis is a syndrome in which the body's immune system overreacts to infection. There may be several life-threatening complications. One of them is acute respiratory distress syndrome (ARDS). ${ }^{1,2}$ ARDS is an acute diffuse lung injury with high incidence. It has been shown that the pathophysiological basis of ARDS includes an excessive and protracted systemic inflammation, ${ }^{3}$ but the exact mechanisms remain unknown. The main risk factors that may cause ARDS include drinking, smoking, air pollution, hypoproteinemia, and diabetes mellitus. ${ }^{4-6}$ Currently, urgent treatment
Correspondence: Junyu Lu; Jianfeng Zhang The Second Affiliated Hospital of Guangxi Medical University, No. 166 Daxuedong Road, Nanning, Guangxi, 530007, People's Republic of China

Tel +8677 I3277I 66

Fax +8677I 3277285

Email junyulu@gxmu.edu.cn;

drzhangjf@163.com 
strategies mainly include low tidal mechanical ventilation, but the outcome is very poor. ${ }^{3}$ Although some progress has been made in treatment options in recent years, the mortality rate remains high. ${ }^{7}$

Previous studies have identified some genes used as biomarkers for ARDS. The high expression of interleukin (IL)-33 can elevate the expression of matrix metallopeptidases (MMP) 2 and 9 in patients with acute lung injury. ${ }^{8}$ SLC2A6 may be a critical biomarker for predicting survival of sepsis patients. ${ }^{9}$ V-set immunoglobulin-domaincontaining 4 (VSIG4) may be involved in lung injury by inducing phagocytosis. ${ }^{10}$ Activation of ERK1/2 by NGF1B depends on MAPKKK c-Raf, eventually inducing inflammation. The NGF1B in the cytoplasm may be involved in the occurrence and development of ARDS. ${ }^{11}$ Down-regulation of the long non-coding RNA GAS5 decreases the expression of angiotensin-converting enzyme 2 (ACE 2), leading to increased levels of microRNA (miR) miR-200c-3p, which promotes the progression of ARDS. ${ }^{12}$ MYC and STAT3 may be the key regulatory genes in the underlying dysfunction of sepsisinduced ARDS. ${ }^{13}$ In addition, a previous study showed that gene set variation index can be used the potential diagnostic tool for sepsis. ${ }^{14}$ And gene set variation index may be considered as the biomarker of bacterial and fungal sepsis. ${ }^{15}$ However, we found that a majority of previous studies mostly focused on single genes or molecules for ARDS. There are only a few studies involving in a set of genes related to ARDS.

Several studies confirmed that alveolar macrophage transcriptional programs are associated with ARDS. ${ }^{16-18}$ We hypothesized that these alveolar macrophage-related ARDS-specific transcriptional programs may be also reflected in blood cells. To investigate our hypothesis in the present study, we identified genes upregulated both in alveolar macrophages and blood cells and constructed a corresponding protein-protein interaction network associated with host response to ADRS. We found that eight genes formed a core gene set, and that the core gene set variation score may be used as a circulating biomarker for ARDS.

\section{Materials and Methods}

\section{Data Collection and Processing}

The data was downloaded from the Gene Expression Omnibus (GEO) database (https://www.ncbi.nlm.nih.gov/ geo/): GSE116560, ${ }^{16}$ GSE89953, ${ }^{19}$ GSE76293 ${ }^{20}$ and
GSE32707. ${ }^{21}$ GSE116560 and GSE89953 were based on GPL6883. GSE116560 was obtained from alveolar macrophages of 68 ARDS samples, while GSE89953 was obtained from peripheral blood monocytes of 26 ARDS samples. The two datasets were combined and then batch effects were removed using the sva routine in $\mathrm{R}^{22}$ From GSE76293, based on GPL570, the gene profiles of neutrophils from 12 ARDS samples and 12 healthy controls were extracted for subsequent analysis. From GSE32707, based on GPL10558, the gene profiles of whole blood cells were used to perform the subsequent analysis, from 18 ARDS samples and 34 healthy controls. The data were normalized using the "normalizeBetweenArrays" function of the limma routine ${ }^{23}$ in $\mathrm{R}$. The probe set was converted into gene symbols, according to the annotation information of the three platforms. The workflow of the study is shown in Figure 1.

\section{Principal Component Analysis (PCA) and Screening Differentially Expressed Genes (DEGs)}

The ggbiplot routine ${ }^{24}$ in $\mathrm{R}$ was applied to perform the PCA. ${ }^{25}$ In GPL6883 datasets (GSE116560 and GSE89953), the DEGs in alveolar macrophages were screened in comparison with peripheral blood monocytes. These DEGs may reflect the response of host monocyte-macrophages to ARDS. ${ }^{18}$ In GSE76293, DEGs in ARDS neutrophils were screened compared to healthy controls, since neutrophils are the most frequent nucleated cell in whole blood. In GSE32707, we screened the DEGs in whole blood between ARDS patients and healthy controls. A total of the DEGs were screened using the limma routine in $\mathrm{R}$. The cut-off criteria are a false discovery rate (FDR)-adjusted $\mathrm{P}<0.05$ and $\mid \log 2$ (fold-change) $\mid>1$. In the study, the common upregulated genes were defined as circulating ARDS alveolar macrophage-related genes (cARDSAMGs).

\section{Gene Ontology (GO) and Kyoto Encyclopedia of Genes and Genomes (KEGG) Pathway Enrichment Analysis}

To explore the potential biological functions of the cARDSAMGs, GO and KEGG pathway enrichment analysis were performed using the clusterProfiler routine in $\mathrm{R}^{26} \mathrm{GO}$ and KEGG networks were clustered using the plug-in ClueGO ${ }^{26}$ in Cytoscape software. ${ }^{27}$ Differences associated with an FDR-adjusted $\mathrm{P}<0.05$ was considered statistically significant. 


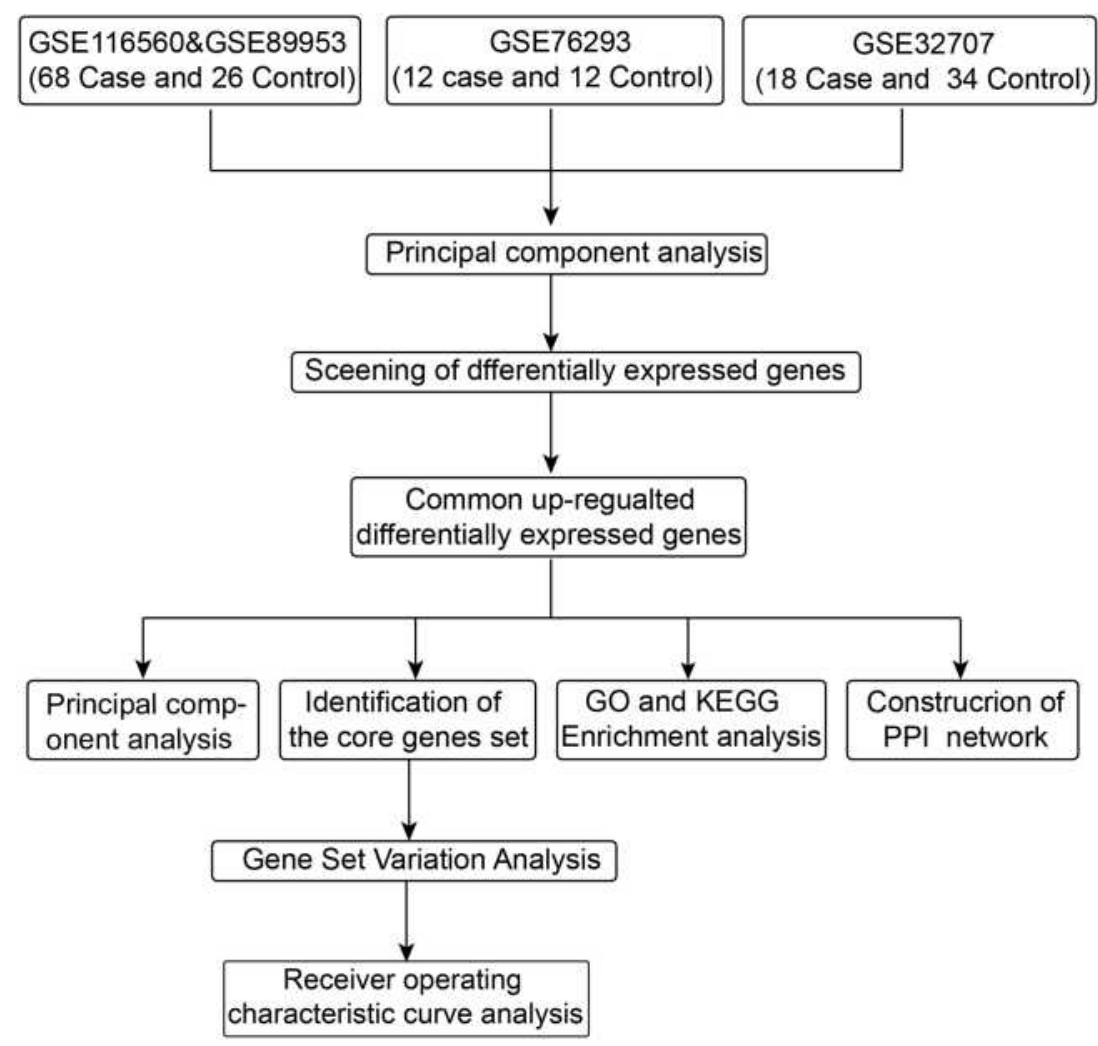

Figure I Workflow chart of the study.

\section{Analysis of Core Genes}

Using STRING database ${ }^{28}$ (https://string-db.org/) and KEGG pathway enrichment analysis, a protein-protein interactions (PPIs) network was constructed for the cARDSAMGs. If genes were involved in ARDSrelated pathways of interest, we defined them as core genes in the network. The GOsemsim routine in $\mathrm{R}^{29}$ was applied to analyze the semantic similarities among GO terms for the cARDSAMGs, applying the following formula: $\mathrm{Pi}=-\lg (\mathrm{p}) * \mid \log 2$ (fold-change) $\mid$, weight $(\mathrm{W})$ value $=$ semantic similarities $*$ mean value of Pi.

\section{Gene Set Variation Analysis (GSVA) and Receiver Operating Characteristic (ROC) Curve Analysis}

The GSVA routine inR ${ }^{30}$ was applied to calculate the core gene set variation analysis (CGSVA) score for individual samples to assess the concerted functional behavior of the core gene set. To further evaluate the potential diagnostic value of the CGSVA score for ARDS, an ROC curve analysis was performed using the $\mathrm{pROC}$ routine in $\mathrm{R}^{31}$

\section{Results \\ Multiple Genes Identified as cARDSAMGs}

PCA showed that global gene expression patterns could distinguish ARDS from controls in GSE116560, GSE89953 and GSE76293, but not in GSE32707 (Figure S1). There were 8522 DEGs in GSE116560 and GSE89953, 6064 in GSE76293, and 7713 in GSE32707 (Figure 2A). A set of 60 DEGs was overexpressed in all the datasets, defined as cARDSAMGs (Figure 2B). Moreover, we obtained the $\mathrm{p}$. value and rank of 60 cARDSAMGs in above three datasets and the ranking based on the p.value (Table S1). PCA showed that the expression patterns of the cARDSAMGs could not distinguish ARDS from healthy controls (Figure $\underline{\mathrm{S} 2}$ ). However, we found that cARDSAMGs can better distinguish ARDS from control compared with the global gene expression patterns (Figures S1 and S2).

\section{Biological Significance of cARDSAMGs}

GO enrichment analysis showed that changes in expression involved mainly genes related to metabolism- and inflammation-related biological processes (Figure 3A). KEGG result showed that DEGs were mainly involved in pathways related 

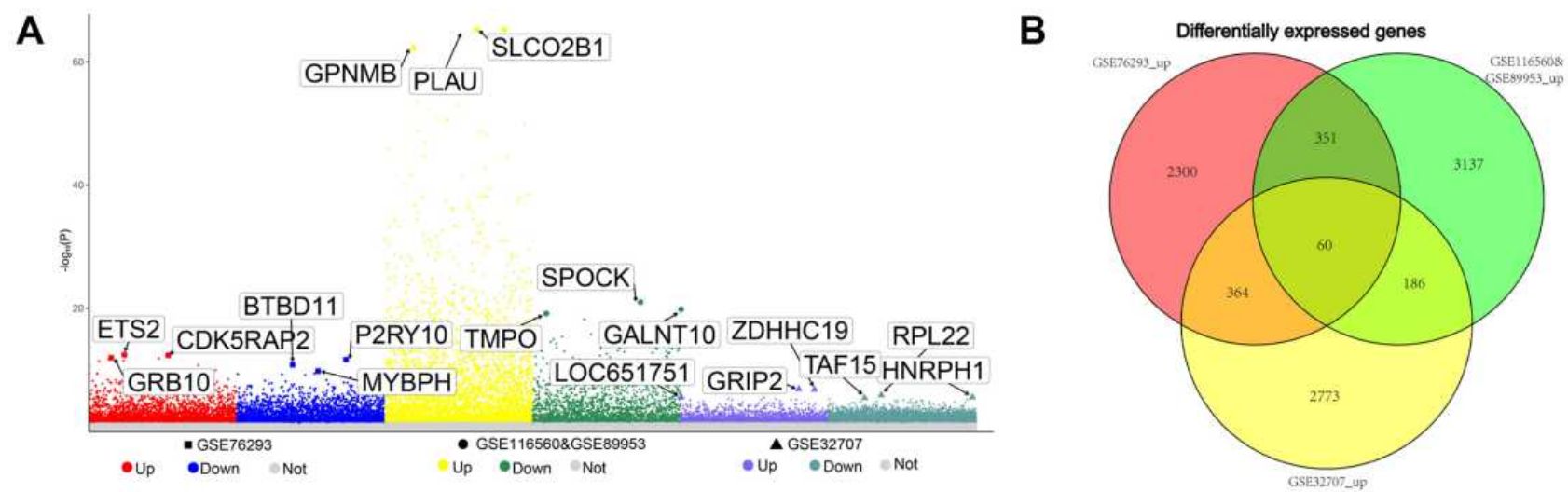

Figure 2 Multiple genes identified as cARDSAMGs. (A) Manhattan plot for DEGs. The three most significantly overexpressed genes are marked in yellow and labeled with their names. (B) Venn diagram to identify upregulated DEGs common to datasets from the three platforms.

Abbreviation: DEGs, differentially expressed genes.

to ARDS, such as Notch the tumor necrosis factor (TNF) and the tumor necrosis factor (TNF) signaling pathways, as well as complement and coagulation cascades (Figure 3B). In addition, we performed the enrichment analysis using the 60 top ranked up-regulated genes in each of the datasets. As shown in Figure S3, we found that the biological processes (BPs) and KEGG pathways were different in each of datasets. But most of them were related to immunity, inflammation and phagocytosis. Furthermore, we randomly selected 60 genes in the three datasets and thereby calculated the enriched GO term. We found that the enriched GO terms mostly were not significantly related with inflammation, immunity and phagocytosis, which further evidenced that the gene set-cARDAMGs was not simply a randomly overlapping set (Figure S4). The ClueGo analysis indicated that DEGs were clustered mainly in biological processes, such as bone resorption, collagen catabolic process, regulation of cartilage development, positive regulation of ATP biosynthetic process, negative regulation of IL-2 production and positive regulation of animal organ morphogenesis (Figure 3C). No significant clustering of KEGG pathways was observed, based on a threshold of FDR-adjusted $\mathrm{P}<0.05$.

\section{Core Genes in the PPIs Network of ARDS}

We constructed the PPIs network of cARDSAMGs based on the STRING database. There were 25 nodes and 189 interaction pairs (Figure 4A). Previous studies showed that some of the biological processes and pathways were related to ARDS, including immunity, inflammation and phagocytosis. $^{32,33}$ Therefore, we selected the following ARDS-related pathways as pathways of interest: Notch,
TNF and IL-17 signaling pathways, as well as complement and coagulation cascades. Subsequently, we identified eight cARDSAMGs as core genes involved in these pathways. The core genes included PTCRA, JAG1, C1QB, ADAM17, C1QA, MMP9, VSIG4 and TNFAIP3. In the complement and coagulation cascades, antigen-antibody complexes act on C1QA and C1QB. They further act on VSIG4, thereby inducing phagocytosis. ${ }^{34,35}$ In the TNF signaling pathway, TNF acts on TNF receptor 1 (TNFR1), ultimately inducing tissue remodeling, autoimmune pathology, neutrophil recruitment and immunity to extracellular pathogens. ${ }^{36,37}$ In the Notch signaling pathway, ADAM17 and JAG1 act on the cell surface receptor Notch, which in turn affects PTCRA. In the TNF signaling pathway, TNF binds to TNFR1, which signals to surface receptors, intracellular signaling and remodeling of extracellular matrix. ${ }^{38,39}$ In the IL-17 signaling pathway, the IL-17 family signals activate downstream pathways that include NF-kappaB, MAPKs and $\mathrm{C} / \mathrm{EBPs}$, which further induces the expression of chemokines, cytokines and antimicrobial peptides ${ }^{40,41}$ (Figure 4B). The semantic similarities among the GO terms of the eight core genes are ranked in Figure $4 \mathrm{C}$, and the $\mathrm{W}$ value of core genes, which may suggest the importance of molecules in this dysfunction, are displayed in Figure 4D.

\section{CGSVA Score May Be Used as a Circulating Biomarker for ARDS}

As shown in Figure S5, the C1QA, C1QB, MMP9, PTCRA and VSIG4 may be serve as circulating diagnostic markers for ARDS. Their areas under the ROC curves (AUCs) were higher than 0.7 in the three datasets. In addition, the CGSVA score was higher in the ARDS group than in the control 


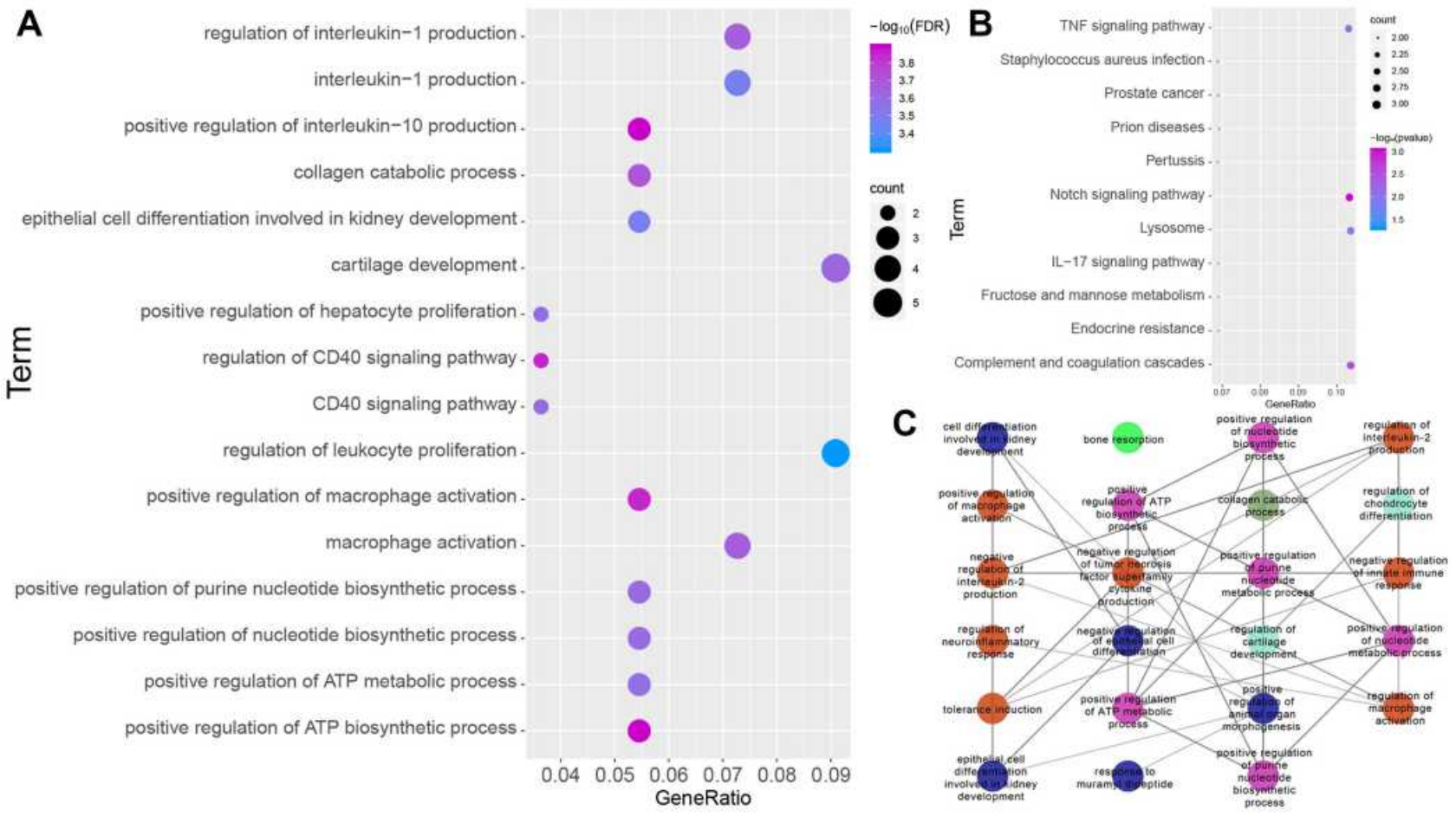

Figure 3 Biological significance of cARDSAMGs. (A) The significantly enriched biological processes, (B) KEGG pathways and (C) GO network in circulating ARDS involving alveolar macrophage-related genes in the three datasets. Different colors of nodes represent different functional groups.

Abbreviations: ARDS, acute respiratory distress syndrome; KEGG, Enrichment in Kyoto Encyclopedia of Genes and Genomes; GO, Gene Ontology.

group in all datasets (Figure 5A). The expression of the core genes is shown in Figure 5A. AUC for CGSVA score was higher than 0.7 in all three datasets, which suggests that the score may be useful as a diagnostic marker for ARDS (Figure 5B). The score in macrophages from the alveolar lavage fluid (GSE116560 and GSE89953) and whole blood (GSE76293 and GSE32707) was higher in ARDS individuals than in healthy controls (Figure 5C).

\section{Discussion}

In this study, the differences in gene expression were explored between whole blood and alveolar lavage in ARDS samples and healthy controls. We selected four gene expression datasets from three platforms, where we found a common set of 60 DEGs upregulated in ARDS patients compared to healthy controls. Some among them have been previously informed to be related to ARDS. For example, immunohistochemical results showed that CD163 is overexpressed in ARDS patients. ${ }^{42}$ The highly expressed IL-33 can drive expression of MMP9 and MMP2 by activating STAT3. The process mainly happens in alveolar macrophages for acute lung injury patients induced by lipopolysaccharide. ${ }^{8}$ Mutations in the ALOX5 gene appear to be most likely cause of interindividual differences in ARDS progression. ${ }^{43}$
Subsequently, we performed a function enrichment analysis for cARDSAMGs. The results showed that these cARDSAMGs were involved mainly in biological processes and pathways related to immunity, inflammation, phagocytosis and nucleic acid turnover. Some of these pathways have been related with ARDS in previous studies. Production of IL-1 and low concentrations of anti-inflammatory cytokines, such as IL-10 and IL-1 receptor antagonist, in bronchoalveolar lavage fluid of early ARDS patients have been closely related to poor prognosis. ${ }^{44}$ Numerous studies have linked inflammation and phagocytosis to ARDS. . $^{32,33,45,46}$

Moreover, we constructed a protein-protein interaction network to analyze the relationship among genes and pathways. We focused on TNF, IL-17 and Notch signaling pathways, as well as complement and coagulation cascades. In complement and coagulation cascades, complement activation may induce the occurrence of phagocytosis, ${ }^{47}$ which plays an essential role in ARDS. ${ }^{48}$ TNF acts as an inflammatory factor, up-regulating the expression of surface receptors, promoting intracellular signaling and remodeling of the extracellular matrix. ${ }^{49}$ Some biomarkers are closely related to ARDS, including surfactant-related proteins and cytokines (IL-1, $-2,-6,-8$, -10 and -15 , and TNF- $\alpha$ ), as well as neutrophil activation 

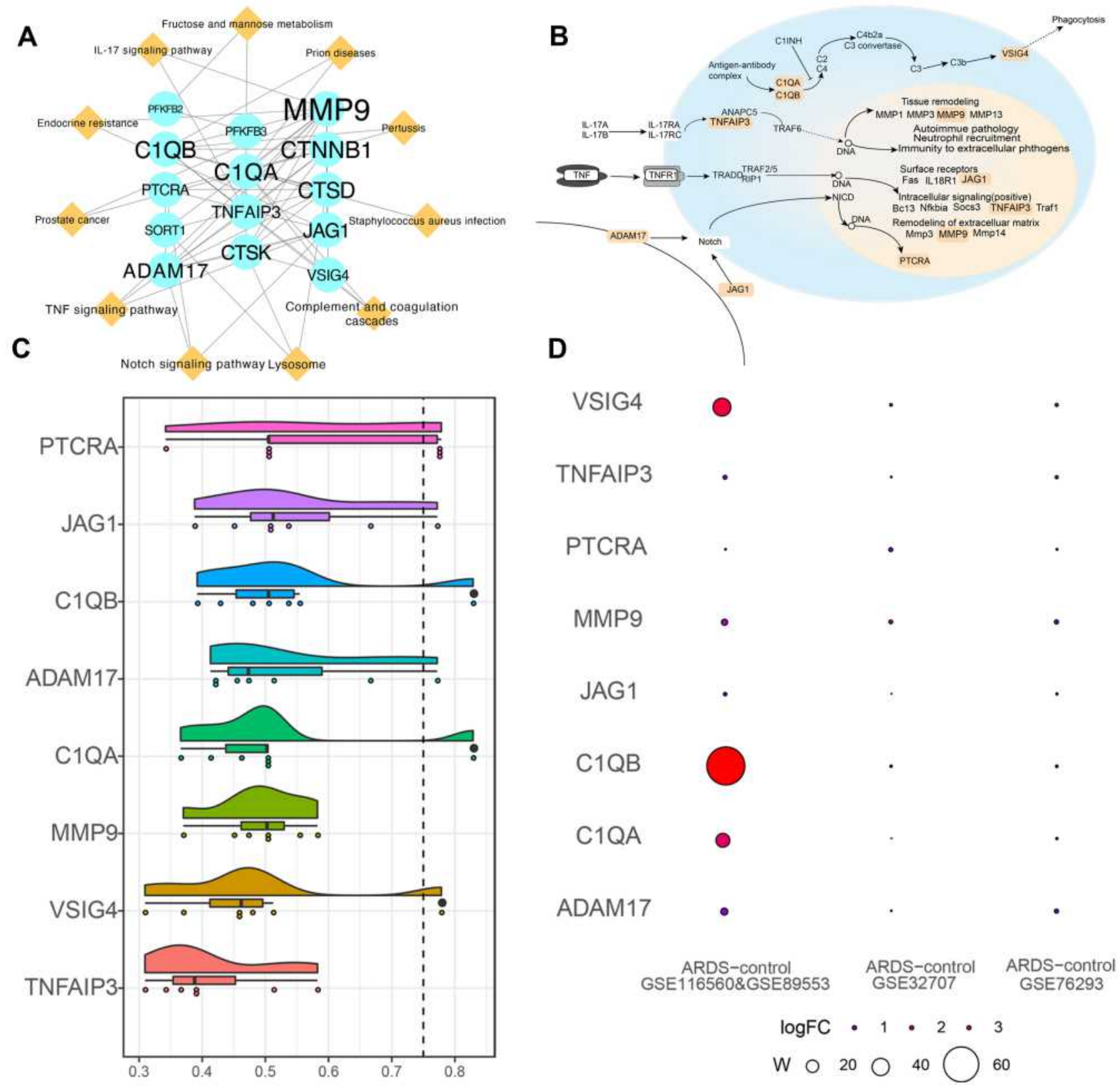

Figure 4 Core genes in the PPIs network of ARDS. (A) PPIs network for alveolar macrophage-related genes in circulating acute respiratory distress syndrome. Ellipses represent genes; diamonds, pathways; and font size, relative degree. (B) Core genes and pathways of interest. (C) Semantic similarities among GO terms for the eight core genes. (D) Weight value of the eight core genes in the three datasets.

Abbreviations: PPIs, protein-protein interactions; GO, Gene Ontology.

markers (MMP9, leukotriene B4 and ferritin). ${ }^{32}$ In the IL17 signaling pathway, IL-17A and IL-17B act through surface receptors IL-17-17RA and IL-17RC to induce immunity and recruit neutrophils. ${ }^{49,50}$ Neutrophil-derived mediators induce epithelial cell death by oxidation of soluble Fas ligand. ${ }^{33,45}$

Eight genes corresponding to the selected pathways were identified as core genes: PTCRA, JAG1, C1QB, ADAM17, C1QA, MMP9, VSIG4 and TNFAIP3. VSIG4 is the main part of phagocytic system: it rapidly removes C3-modified particles. ${ }^{10}$ MMP9 is a secreted zinc metallopeptidase. ${ }^{51}$ MMP9 mediates protein degradation in the extracellular matrix of alveolar epithelial cells, mainly in intercellular junction proteins and proteins that anchor cells to the basement membrane. ${ }^{52}$ In addition, MMP9 is enriched mainly in macrophages and neutrophils, ${ }^{53,54}$ and involved in the occurrence and development of emphysema and asthma. ${ }^{55,56}$ TNFAIP3 was 

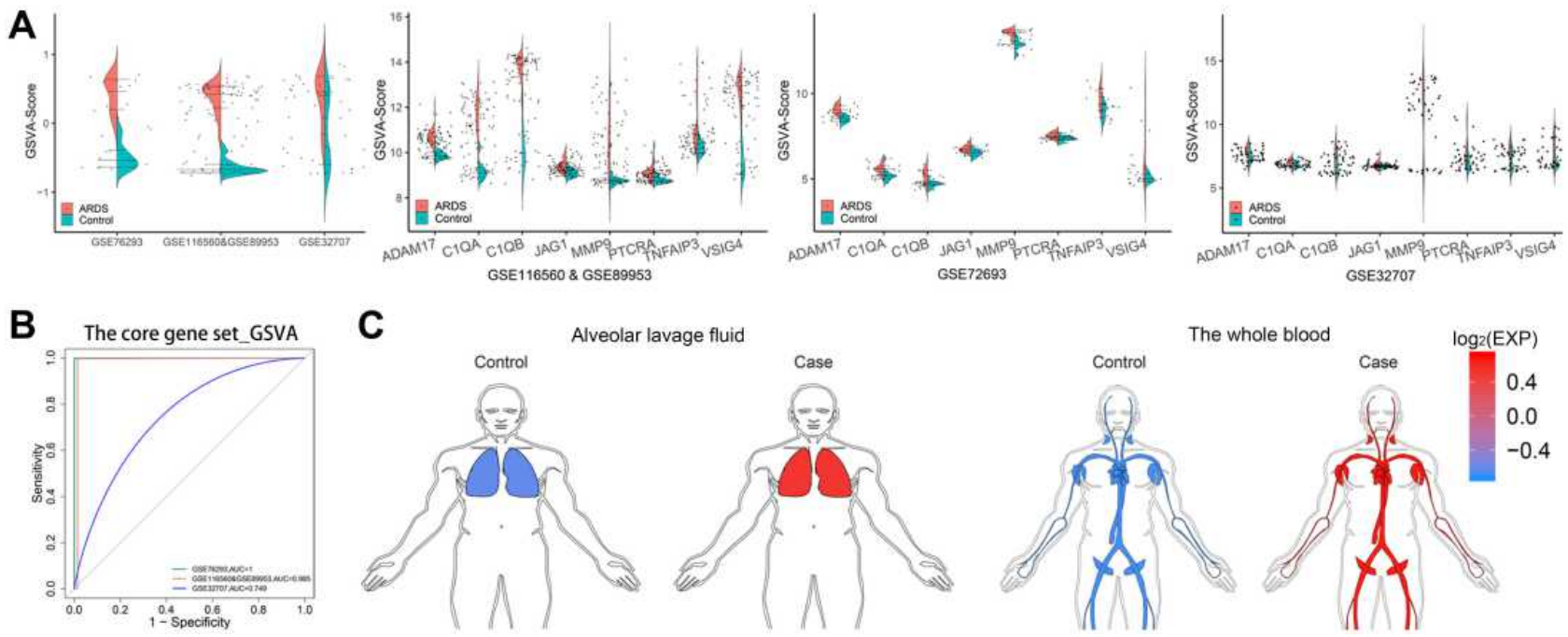

Figure 5 CGSVA score may serve as a circulating biomarker for ARDS. (A) a, GSVA scores of the core gene set in the three datasets. Expression of core genes in (b) GSE76293, (c) GSEI 16560 \& GSE89953 and (d) GSE32707. (B) ROC curves of the gene set GSVA score in the three datasets. (C) The GSVA scores in alveolar whole blood (GSE76293 and GSE32707) and lavage fluid (GSEI 16560 and GSE89953) were higher for patients with ARDS than for controls.

Abbreviations: GSVA, gene set variation analysis; ROC, receiver operating characteristic; ARDS, acute respiratory distress syndrome.

identified as a gene whose expression is rapidly induced by TNF. The protein encoded by this gene has been shown to inhibit nuclear factor (NF)-kappa B activation as well as TNF-mediated apoptosis. ${ }^{57}$ PTCRA encodes a single channel type 1 membrane protein in mature $\mathrm{T}$ cells. This protein forms a T-cell pre-receptor complex together with CD3 and T cell receptor beta chain (TCRB) complexes, thereby regulating the early development of $\mathrm{T}$ cells. ${ }^{58-60}$ C1QB may be associated with the immune response after injury. ${ }^{61}$

In addition, CGSVA score may be used as a biomarker for ARDS patients. Furthermore, the CGSVA scores associated with ARDS both in whole blood and alveolar macrophages were significantly higher in ARDS patients than in healthy controls. This may suggest that these alveolar macrophage-related ARDS-specific transcriptional programs are also reflected in blood cells.

Our study presents several limitations. Firstly, our predictions are based on bioinformatic analyses, and therefore further experimental verification is needed. Secondly, most of alveolar macrophage genes have a dynamic pattern, but we only select genes that are significantly up-regulated in all time point without the consider of time changes. Thirdly, the whole transcriptome sequencing data of macrophages in whole blood and alveolar lavage fluid are difficult to find, currently. Whether these 8 cARDSAMGs may be used as diagnostic markers needs to verify in a larger independent clinical sample. Fourthly, it is shown that the gene set has a diagnostic performance in each of datasets in this study. Therefore, we believe that this gene set is specific for ARDS to some extent. But whether it is specific enough to ARDS, any inflammation or infection, still needs to be further clarified. Finally, we need to further explore the upstream regulators of core genes to reveal in more detail the imbalance network in ARDS.

\section{Conclusions}

We identified a core gene set (PTCRA, JAG1, C1QB, ADAM17, C1QA, MMP9, VSIG4 and TNFAIP3) that may help predict ARDS. The ARDS alveolar macrophagerelated CGSVA score may be helpful for the diagnosis of ARDS.

\section{Data Sharing Statement}

The data was downloaded from the Gene Expression Omnibus (GEO) database (https://www.ncbi.nlm.nih.gov/ geo/): GSE116560, GSE89953, GSE76293 and GSE32707.

\section{Funding}

This study was supported by the National Natural Science Foundation of China (81660132 and 81960343), the Reserve Cadre Training Program Science Foundation of the Second Affiliated Hospital of Guangxi Medical University (No. HBRC201805), Guangxi Health Commission key Laboratory of Emergency and Critical Medicine (The Second Affiliated Hospital of Guangxi 
Medical University) and the High-level Medical Expert Training Program of Guangxi “139" Plan Funding (G201903027).

\section{Disclosure}

The authors declare that the research was conducted in the absence of any commercial or financial relationships that could be construed as a potential conflict of interest.

\section{References}

1. Zeng X, Feng J, Yang Y, et al. Screening of key genes of sepsis and septic shock using bioinformatics analysis. J Inflamm Res. 2021;14:829-841. doi:10.2147/JIR.S301663

2. Feng J, Pang J, He D, et al. Identification of genes with altered methylation and its role in early diagnosis of sepsis-induced acute respiratory distress syndrome. Int J Gen Med. 2021;14:243-253. doi:10.2147/IJGM.S287960

3. Peter JV, John P, Graham PL, Moran JL, George IA, Bersten A. Corticosteroids in the prevention and treatment of acute respiratory distress syndrome (ARDS) in adults: meta-analysis. BMJ. 2008;336 (7651):1006-1009. doi:10.1136/bmj.39537.939039.BE

4. Matthay MA, Zemans RL, Zimmerman GA, et al. Acute respiratory distress syndrome. Nat Rev Dis Primers. 2019;5(1):18. doi:10.1038/ s41572-019-0069-0

5. Ferguson ND, Frutos-Vivar F, Esteban A, et al. Clinical risk conditions for acute lung injury in the intensive care unit and hospital ward: a prospective observational study. Crit Care. 2007;11(5):R96. doi:10.1186/cc6113

6. Agrawal A, Matthay MA, Kangelaris $\mathrm{KN}$, et al. Plasma angiopoietin-2 predicts the onset of acute lung injury in critically ill patients. Am J Respir Crit Care Med. 2013;187(7):736-742. doi:10.1164/rccm.201208-1460OC

7. Bellani G, Laffey JG, Pham T, et al. Epidemiology, patterns of care, and mortality for patients with acute respiratory distress syndrome in intensive care units in 50 countries. JAMA. 2016;315(8):788-800. doi:10.1001/jama.2016.0291

8. Liang Y, Yang N, Pan G, Jin B, Wang S, Ji W. Elevated IL-33 promotes expression of MMP2 and MMP9 via activating STAT3 in alveolar macrophages during LPS-induced acute lung injury. Cell Mol Biol Lett. 2018;23:52. doi:10.1186/s11658-018-0117-x

9. Li Z, Huang B, Yi W, et al. Identification of potential early diagnostic biomarkers of sepsis. J Inflamm Res. 2021;14:621-631. doi:10.2147/ JIR.S298604

10. Helmy KY, Katschke KJ, Gorgani NN, et al. CRIg: a macrophage complement receptor required for phagocytosis of circulating pathogens. Cell. 2006;124(5):915-927. doi:10.1016/j. cell.2005.12.039

11. Jiang Y, Zeng Y, Huang X, et al. Nur77 attenuates endothelin-1 expression via downregulation of NF-kappaB and p38 MAPK in A549 cells and in an ARDS rat model. Am J Physiol Lung Cell Mol Physiol. 2016;311(6):L1023-L1035. doi:10.1152/ ajplung.00043.2016

12. Li HB, Zi PP, Shi HJ, Gao M, Sun RQ. [Role of signaling pathway of long non-coding RNA growth arrest-specific transcript 5/ microRNA-200c-3p/angiotensin converting enzyme 2 in the apoptosis of human lung epithelial cell A549 in acute respiratory distress syndrome]. Zhonghua Yi Xue Za Zhi. 2018;98(41):3354-3359. Chinese. doi:10.3760/cma.j.issn.0376-2491.2018.41.013

13. Zhang J, Luo Y, Wang X, et al. Global transcriptional regulation of STAT3- and MYC-mediated sepsis-induced ARDS. Ther Adv Respir Dis. 2019;13:1753466619879840. doi:10.1177/1753466619879840
14. $\mathrm{Lu} \mathrm{J}, \mathrm{Li} \mathrm{Q}, \mathrm{Wu} \mathrm{Z}$, et al. Two gene set variation indexes as potential diagnostic tool for sepsis. Am J Transl Res. 2020;12(6):2749-2759.

15. Zheng X, Luo Y, Li Q, et al. Two gene set variation index as biomarker of bacterial and fungal sepsis. Biomed Res Int. 2020;2020:8182358. doi:10.1155/2020/8182358

16. Morrell ED, Bhatraju PK, Mikacenic CR, et al. Alveolar macrophage transcriptional programs are associated with outcomes in acute respiratory distress syndrome. Am $J$ Respir Crit Care Med. 2019;200(6):732-741. doi:10.1164/rccm.201807-1381OC

17. Thompson BT, Chambers RC, Liu KD, Drazen JM. Acute respiratory distress syndrome. $N$ Engl $J$ Med. 2017;377(6):562-572. doi:10.1056/NEJMra1608077

18. Aggarwal NR, King LS, D'Alessio FR. Diverse macrophage populations mediate acute lung inflammation and resolution. Am J Physiol Lung Cell Mol Physiol. 2014;306(8):L709-25. doi:10.1152/ ajplung.00341.2013

19. Morrell ED, Radella F, Manicone AM, et al. Peripheral and alveolar cell transcriptional programs are distinct in acute respiratory distress syndrome. Am J Respir Crit Care Med. 2018;197(4):528-532. doi:10.1164/rccm.201703-0614LE

20. Juss JK, House D, Amour A, et al. Acute respiratory distress syndrome neutrophils have a distinct phenotype and are resistant to phosphoinositide 3-kinase inhibition. Am J Respir Crit Care Med. 2016;194(8):961-973. doi:10.1164/rccm.201509-18180C

21. Dolinay T, Kim YS, Howrylak J, et al. Inflammasome-regulated cytokines are critical mediators of acute lung injury. Am J Respir Crit Care Med. 2012;185(11):1225-1234. doi:10.1164/rccm.201201-0003OC

22. Leek JT, Johnson WE, Parker HS, Jaffe AE, Storey JD. The sva package for removing batch effects and other unwanted variation in high-throughput experiments. Bioinformatics. 2012;28(6):882-883. doi:10.1093/bioinformatics/bts034

23. Cui Y, Yang X, Didelot X, et al. Epidemic clones, oceanic gene pools, and eco-LD in the free living marine pathogen vibrio parahaemolyticus. Mol Biol Evol. 2015;32(6):1396-1410. doi: 10.1093/molbev/msv009

24. de Oliveira LA, da Silva CP, Nuvunga JJ, da Silva AQ, Balestre M. Bayesian GGE biplot models applied to maize multi-environments trials. Genet Mol Res. 2016;15(2). doi:10.4238/gmr.15028612

25. David CC, Jacobs DJ. Principal component analysis: a method for determining the essential dynamics of proteins. Methods Mol Biol. 2014;1084:193-226. doi:10.1007/978-1-62703-658-0_11

26. Yu G, Wang LG, Han Y, He QY. clusterProfiler: an R package for comparing biological themes among gene clusters. OMICS. 2012;16 (5):284-287. doi:10.1089/omi.2011.0118

27. Kohl M, Wiese S, Warscheid B. Cytoscape: software for visualization and analysis of biological networks. Methods Mol Biol. 2011;696:291-303. doi:10.1007/978-1-60761-987-1_18

28. Szklarczyk D, Gable AL, Lyon D, et al. STRING v11: protein-protein association networks with increased coverage, supporting functional discovery in genome-wide experimental datasets. Nucleic Acids Res. 2019;47(D1):D607-D613. doi:10.1093/nar/gky1131

29. Yu G, Li F, Qin Y, Bo X, Wu Y, Wang S. GOSemSim: an R package for measuring semantic similarity among GO terms and gene products. Bioinformatics. 2010;26(7):976-978. doi:10.1093/bioinformatics/btq064

30. Hanzelmann S, Castelo R, Guinney J. GSVA: gene set variation analysis for microarray and RNA-seq data. BMC Bioinform. 2013;14:7. doi:10.1186/1471-2105-14-7

31. Robin X, Turck N, Hainard A, et al. pROC: an open-source package for $\mathrm{R}$ and $\mathrm{S}+$ to analyze and compare ROC curves. BMC Bioinform. 2011;12:77. doi:10.1186/1471-2105-12-77

32. Sun X, Ma SF, Wade MS, et al. Functional promoter variants in sphingosine 1-phosphate receptor 3 associate with susceptibility to sepsis-associated acute respiratory distress syndrome. Am J Physiol Lung Cell Mol Physiol. 2013;305(7):L467-77. doi:10.1152/ ajplung.00010.2013 
33. Hogner K, Wolff T, Pleschka S, et al. Macrophage-expressed IFN-beta contributes to apoptotic alveolar epithelial cell injury in severe influenza virus pneumonia. PLoS Pathog. 2013;9(2): e1003188. doi:10.1371/journal.ppat.1003188

34. Bajic G, Degn SE, Thiel S, Andersen GR. Complement activation, regulation, and molecular basis for complement-related diseases. EMBO J. 2015;34(22):2735-2757. doi:10.15252/embj.201591881

35. Mathern DR, Heeger PS. Molecules great and small: the complement system. Clin J Am Soc Nephrol. 2015;10(9):1636-1650. doi:10.2215/ CJN.06230614

36. Chu WM. Tumor necrosis factor. Cancer Lett. 2013;328(2):222-225. doi:10.1016/j.canlet.2012.10.014

37. Shuh M, Bohorquez H, Loss GE, Cohen AJ. Tumor necrosis factor-alpha: life and death of hepatocytes during liver ischemia/ reperfusion injury. Ochsner J. 2013;13(1):119-130.

38. Hansson EM, Lendahl U, Chapman G. Notch signaling in development and disease. Semin Cancer Biol. 2004;14(5):320-328. doi:10.1016/j.semcancer.2004.04.011

39. Callahan R, Egan SE. Notch signaling in mammary development and oncogenesis. J Mammary Gland Biol Neoplasia. 2004;9(2):145-163. doi:10.1023/B:JOMG.0000037159.63644.81

40. Zenobia C, Hajishengallis G. Basic biology and role of interleukin-17 in immunity and inflammation. Periodontol 2000. 2015;69 (1):142-159. doi:10.1111/prd.12083

41. Gaffen SL, Jain R, Garg AV, Cua DJ. The IL-23-IL-17 immune axis: from mechanisms to therapeutic testing. Nat Rev Immunol. 2014;14 (9):585-600. doi:10.1038/nri3707

42. Maretta M, Toth S, Jonecova Z, et al. Immunohistochemical expression of MPO, CD163 and VEGF in inflammatory cells in acute respiratory distress syndrome: a case report. Int J Clin Exp Pathol. 2014;7(7):4539-4544.

43. Geiger EV, Doehring A, Kirchhof A, Lotsch J. Functional variants of the human 5-lipoxygenase gene and their genetic diagnosis. Prostaglandins Leukot Essent Fatty Acids. 2009;80(5-6):255-262. doi:10.1016/j.plefa.2009.04.001

44. Donnelly SC, Strieter RM, Reid PT, et al. The association between mortality rates and decreased concentrations of interleukin-10 and interleukin-1 receptor antagonist in the lung fluids of patients with the adult respiratory distress syndrome. Ann Intern Med. 1996;125 (3):191-196. doi:10.7326/0003-4819-125-3-199608010-00005

45. Johnson ER, Matthay MA. Acute lung injury: epidemiology, pathogenesis, and treatment. J Aerosol Med Pulm Drug Deliv. 2010;23 (4):243-252. doi:10.1089/jamp.2009.0775

46. Matthay MA, Ware LB, Zimmerman GA. The acute respiratory distress syndrome. J Clin Invest. 2012;122(8):2731-2740. doi:10.1172/JCI60331

47. Zilow G, Sturm JA, Rother U, Kirschfink M. Complement activation and the prognostic value of $\mathrm{C} 3 \mathrm{a}$ in patients at risk of adult respiratory distress syndrome. Clin Exp Immunol. 1990;79(2):151-157. doi:10.1111/j.1365-2249.1990.tb05171.x

48. Hammerschmidt DE, Weaver LJ, Hudson LD, Craddock PR, Jacob HS. Association of complement activation and elevated plasma-C5a with adult respiratory distress syndrome. Pathophysiological relevance and possible prognostic value. Lancet. 1980;1(8175):947-949. doi:10.1016/s0140-6736(80)91403-8
49. Roca FJ, Whitworth LJ, Redmond S, Jones AA, Ramakrishnan L. $\mathrm{TNF}$ induces pathogenic programmed macrophage necrosis in tuberculosis through a mitochondrial-lysosomal-endoplasmic reticulum circuit. Cell. 2019;178(6):1344-1361 el1. doi:10.1016/j. cell.2019.08.004

50. Doyle MS, Collins ES, FitzGerald OM, Pennington SR. New insight into the functions of the interleukin-17 receptor adaptor protein Act1 in psoriatic arthritis. Arthritis Res Ther. 2012;14(5):226. doi:10.1186/ ar4071

51. Nagase H, Barrett AJ, Woessner JF. Nomenclature and glossary of the matrix metalloproteinases. Matrix Suppl. 1992;1:421-424.

52. O'Kane CM, McKeown SW, Perkins GD, et al. Salbutamol up-regulates matrix metalloproteinase-9 in the alveolar space in the acute respiratory distress syndrome. Crit Care Med. 2009;37 (7):2242-2249. doi:10.1097/CCM.0b013e3181a5506c

53. Kelly EA, Busse WW, Jarjour NN. Increased matrix metalloproteinase-9 in the airway after allergen challenge. Am J Respir Crit Care Med. 2000;162(3):1157-1161. doi:10.1164/ ajrccm.162.3.9908016

54. Coussens LM, Tinkle CL, Hanahan D, Werb Z. MMP-9 supplied by bone marrow-derived cells contributes to skin carcinogenesis. Cell. 2000;103(3):481-490. doi:10.1016/s0092-8674(00)00139-2

55. Nakashima K, Hirota T, Obara K, et al. A functional polymorphism in MMP-9 is associated with childhood atopic asthma. Biochem Biophys Res Commun. 2006;344(1):300-307. doi:10.1016/j. bbrc.2006.03.102

56. Minematsu N, Nakamura H, Tateno H, Nakajima T, Yamaguchi K. Genetic polymorphism in matrix metalloproteinase-9 and pulmonary emphysema. Biochem Biophys Res Commun. 2001;289(1):116-119. doi:10.1006/bbrc.2001.5936

57. Das S, Pandey K, Kumar A, et al. TGF-beta1 re-programs TLR4 signaling in L. donovani infection: enhancement of SHP-1 and ubiquitin-editing enzyme A20. Immunol Cell Biol. 2012;90 (6):640-654. doi:10.1038/icb.2011.80

58. Aifantis I, Borowski C, Gounari F, Lacorazza HD, Nikolich-Zugich J, von Boehmer H. A critical role for the cytoplasmic tail of pTalpha in T lymphocyte development. Nat Immunol. 2002;3(5):483-488. doi:10.1038/ni779

59. Del Porto P, Bruno L, Mattei MG, von Boehmer H, Saint-Ruf C. Cloning and comparative analysis of the human pre-T-cell receptor alpha-chain gene. Proc Natl Acad Sci U S A. 1995;92 (26):12105-12109. doi:10.1073/pnas.92.26.12105

60. Saint-Ruf C, Lechner O, Feinberg J, von Boehmer H. Genomic structure of the human pre-T cell receptor alpha chain and expression of two mRNA isoforms. Eur J Immunol. 1998;28(11):3824-3831. doi:10.1002/(SICI)1521-4141(199811)28:11<3824::AIDIMMU3824>3.0.CO;2-9

61. Byrnes KR, Garay J, Di Giovanni S, et al. Expression of two temporally distinct microglia-related gene clusters after spinal cord injury. Glia. 2006;53(4):420-433. doi:10.1002/glia.20295
Journal of Inflammation Research

\section{Publish your work in this journal}

The Journal of Inflammation Research is an international, peerreviewed open-access journal that welcomes laboratory and clinical findings on the molecular basis, cell biology and pharmacology of inflammation including original research, reviews, symposium reports, hypothesis formation and commentaries on: acute/chronic inflammation; mediators of inflammation; cellular processes; molecular mechanisms; pharmacology and novel anti-inflammatory drugs; clinical conditions involving inflammation. The manuscript management system is completely online and includes a very quick and fair peerreview system. Visit http://www.dovepress.com/testimonials.php to read real quotes from published authors. 